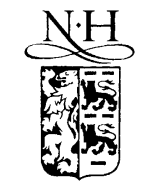

ELSEVIER

\title{
Relaxation of dynamically correlated clusters
}

\author{
Agnieszka Jurlewicz ${ }^{\mathrm{a}}$, Karina Weron ${ }^{\mathrm{b}, *}$ \\ ${ }^{a}$ Institute of Mathematics, Wrockaw University of Technology, Wyb. Wyspiańskiego 27, 50-370 Wroctaw, Poland \\ ${ }^{\mathrm{b}}$ Institute of Physics, Wroclaw University of Technology, Wyb. Wyspiańskiego 27, 50-370 Wroctaw, Poland
}

\begin{abstract}
In the frame of a new probabilistic approach to relaxation, the scenario of relaxation leading to the HavriliakNegami and Kohlrausch-Williams-Watts responses of complex systems is presented. In this approach the macroscopic laws are related to the micro/mesoscopic stochastic characteristics of the relaxing systems. This provides a rigorous formulation of the energy-criterion argument, introduced by Jonscher to explain the commonly observed highfrequency fractional power law. The presented considerations reinforce the physical significance of the empirically found forms of relaxation, and open a new line of analysis of relaxation phenomena. (c) 2002 Elsevier Science B.V. All rights reserved.
\end{abstract}

\section{Introduction}

When subjected to an external perturbation, the subsequent relaxation of condensed matter is usually observed to proceed in a non-exponential fashion. A class of the systems exhibiting this kind of behavior includes various complex materials as supercooled liquids, amorphous polymers, and disordered crystals (see e.g. [1-5]).

The dielectric response of such different materials is characterized well enough by a few empirical functions, and a common property [3] of those functions is that they exhibit the high-frequency power law in the complex dielectric susceptibility $\chi(\omega)$

\footnotetext{
${ }^{*}$ Corresponding author. Fax: +48-71 227751.

E-mail addresses: a.jurlewicz@im.pwr.wroc.pl (A. Jurlewicz), karina@rainbow.if.pwr.wroc.pl (K. Weron).
}

$\chi(\omega) \propto\left(\frac{\mathrm{i} \omega}{\omega_{\mathrm{p}}}\right)^{n-1}$ for $\omega \gg \omega_{\mathrm{p}}$,

where the exponent $n$ falls in the range $(0,1)$ and the constant $\omega_{\mathrm{p}}$ is the loss peak frequency.

In theoretical attempts to model relaxation it is unanimously assumed that the observed relaxation laws correspond to a kind of general behavior which is independent of the details of examined systems. In the framework of statistical models this idea is intuitively justified since for large scale behavior of complex systems one expects 'averaging principles' like the law of large numbers to be in force. However, it is very difficult to make this intuition precise in concrete examples. In general, the relaxation behavior of the complex system as a whole cannot be attributed to any particular object chosen from those forming the system, and the problem of construction of an 'averaged' object 
representing the entire system in relaxation processes is not a trivial one. The information on the stochastic micro/mesoscopic scenario which relates the local random characteristics of complex systems to the deterministic and universally valid empirical relaxation laws is of a great importance.

The most basic question to answer is whether the observed dynamical complexity is intrinsic (with all regions of the sample exhibiting a similar non-exponential response) or whether the nonexponential net behavior is a result of heterogeneity (with localized degrees of freedom relaxing exponentially with randomly distributed relaxation times or rates) (see e.g. [6-15]). Considerable experimental evidence indicates that the relaxation response is often influenced by heterogeneity. Several studies of different systems, including supercooled liquids near their calorimetric glass transition temperature, have shown that heterogeneity occurs on length scales of 1-10 nm [16-19]. The NMR measurements have led to the conclusion that although the appearing domains are not static, their distinct relaxation rates persist long enough to cause the broadened, non-exponential response [20,21]. The dynamic heterogeneity, which evolves as the sample responds, has been also observed by means of the dielectric spectral hole burning technique. This most direct technique for investigating the constituents in the net response of a macroscopic sample has established that the non-exponential dielectric response clearly results from a relaxation-rate distribution of independently relaxing randomly sized mesoscopic regions [22,23].

On the other hand, the recent advances in the stochastic theory of relaxation [24-27] have provided the technique to formulate both the micro/ mesoscopic scenario of relaxation and the resulting effective representation of the system. The approach, which is based on the general probabilistic formalism of limit theorems, enables us to treat relaxation of complex systems regardless of the precise nature of local interactions. It has the advantage of clarifying the nature of relaxation phenomena despite the difficulties caused by the use of a new (going beyond the classical methods of statistical physics) language to describe the time evolution of the non-equilibrium state of a stochastic system.

The fundamental consequence of property (1) is that for large $\omega$ the ratio of the imaginary to real components of the complex susceptibility $\chi(\omega)=$ $\chi^{\prime}(\omega)-\mathrm{i} \chi^{\prime \prime}(\omega)$ becomes a constant dependent only on the exponent $n$ :

$\frac{\chi^{\prime \prime}(\omega)}{\chi^{\prime}(\omega)}=\cot \left(n \frac{\pi}{2}\right)$ for $\omega \gg \omega_{\mathrm{p}}$.

The physical interpretation of (2) is that at high frequencies the ratio of the macroscopic energy lost per radian to the energy stored at the peak is independent of frequency. Jonscher alleged [1,5] that fractional power law (1) and relation (2) are inescapably connected with the fact that the energy loss in every microscopic reversal is independent of the rate of reversals in the corresponding frequency range. He assumed that since in any dielectric system the total polarization is the sum of individual microscopic polarizations and the total loss is the sum of individual microscopic losses, the microscopic relationship also must posses property (2) with the same exponent $n$. The limitation of the above energy-criterion argument is the lack of precise derivation. However, in our opinion, a theoretical model that includes the resulting from physical intuition frequency-independent microscopic energy relation can open a new line of analysis of the dielectric relaxation and indicate the properties of complex stochastic systems.

In this paper we present stochastic description of relaxation mechanism, introduced in [28]. In the proposed approach the microscopic stochastic scenario of relaxation yields the macroscopic relaxation response of the Kohlrausch-WilliamsWatts (KWW) and Havriliak-Negami (HN) forms and, under very general conditions, provides the energy criterion of the form (2) on the micro/meso/ macroscopic levels. The relation between the phenomenological relaxation laws and the microscopic cause is discussed from the background of the statistical properties of constituents in the net response of a macroscopic sample, and the energy criterion argument is involved by the spatio-temporal scaling properties of the mesoscopic cooperative regions of dynamically correlated clusters. 


\section{Probabilistic representation of the empirical relaxation functions}

It is well known that the non-exponential timedomain relaxation function $\phi(t)$ has to fulfill the two-state master equation

$\frac{\mathrm{d} \phi(t)}{\mathrm{d} t}=-r(t) \phi(t), \quad \phi(0)=1$,

where the non-negative, time-dependent quantity $r(t)$ is the transition rate of the relaxing system (i.e. the probability of transition per unit time), see e.g. [8]. The inverse Stieltjes-Fourier transform

$\phi^{*}(\omega)=\int_{0}^{\infty} \mathrm{e}^{-\mathrm{i} t \omega} \mathrm{d}(1-\phi(t))$

relates the time-domain response $\phi(t)$ to the complex susceptibility $\chi(\omega)$ by the formula $\chi(\omega)=$ $\phi^{*}(\omega)\left(\chi_{0}-\chi_{\infty}\right)+\chi_{\infty}$, where the constant $\chi_{\infty}$ represents the asymptotic value of the dielectric susceptibility $\chi(\omega)$ at high frequencies, and $\chi_{0}$ is the value of the opposite limit.

In general, the solution of Eq. (3) has the meaning of the survival probability of the nonequilibrium initial state of the relaxing system [29]. Its value $\phi(t)$ is determined by the probability that the system as a whole will not make a transition out of its original state for at least time $t$ after entering it at $t=0$. Introducing $\tilde{\theta}$, the random waiting time of the system for the transition from its initial state, one obtains probabilistic form of the relaxation function

$\phi(t)=\operatorname{Pr}(\tilde{\theta} \geqslant t)$.

The frequency-domain response $\phi^{*}(\omega)$, given by (4), can be expressed by $\tilde{\theta}$ as well; namely, $\phi^{*}(\omega)=$ $\left\langle\mathrm{e}^{-\mathrm{i} \omega \tilde{\theta}}\right\rangle$, where $\langle\cdot\rangle$ denotes the expected value.

On the other hand, following the historically oldest approach to relaxation, the non-exponential relaxation function $\phi(t)$ is assumed to take the form of a weighted average of an exponential decay $\mathrm{e}^{-t / \tau}$ with respect to the distribution $w(\tau) \mathrm{d} \tau$ of relaxation time $\tau$ [2]. Therefore, $\phi(t)$ can be represented by a random variable $\tilde{T}$ distributed as the considered relaxation time, or equivalently, by $\tilde{\beta}=1 / \tilde{T}$, representing the corresponding relaxation rate. Namely,

$$
\begin{aligned}
\phi(t) & =\int_{0}^{\infty} \mathrm{e}^{-t / \tau} w(\tau) \mathrm{d} \tau=\left\langle\mathrm{e}^{-t / \tilde{T}}\right\rangle=\left\langle\mathrm{e}^{-t \tilde{\beta}}\right\rangle \\
& =\int_{0}^{\infty} \mathrm{e}^{-t b} g(b) \mathrm{d} b .
\end{aligned}
$$

Formula (6) assigns the relaxation function $\phi(t)$ to the Laplace transform of the relaxation-rate distribution $g(b) \mathrm{d} b$. The probability density functions $w(\tau)$ and $g(b)$ are related to each other, $g(b)=b^{-2} w\left(b^{-1}\right)$.

Summing up, any relaxation function $\phi(t)$, being a solution of two-state master Eq. (3), can be associated with $\tilde{\theta}$, the waiting time of the system for the transition from the initial state; $\tilde{T}$, the relaxation time; and $\tilde{\beta}$, the relaxation rate of the system. Both $\phi(t)$ and $\phi^{*}(\omega)$ can be unequivocally represented by each of these random variables. Moreover, the random variables $\tilde{\theta}$ and $\tilde{\beta}$ (or $\tilde{T}=1 / \tilde{\beta})$ are strictly related by means of $\phi(t)$ since

$\phi(t)=\operatorname{Pr}(\tilde{\theta} \geqslant t)=\left\langle\mathrm{e}^{-t \tilde{\beta}}\right\rangle$.

Let us add that although the language of random variables seems to be abstract and artificial here, it gives a new possibility for modeling of relaxation phenomena as shall be shown in the next sections.

Among the empirical relaxation functions, commonly used to fit the dielectric data, the most popular analytical expression applied to the frequency-domain measurements is given by the $\mathrm{HN}$ relaxation function [2-5]

$\phi_{\mathrm{HN}}^{*}(\omega)=\frac{1}{\left(1+\left(\mathrm{i} \omega / \omega_{\mathrm{p}}\right)^{\alpha}\right)^{\gamma}}$,

where $0<\alpha, \gamma<1$. For $\alpha=1$ and $\gamma<1$, Eq. (8) takes the form known as the Cole-Davidson (CD) function; for $\gamma=1$ and $\alpha<1$ it takes the form of the Cole-Cole (CC) function, and for $\alpha=1$ and $\gamma=1$ one obtains the classical Debye (D) form. Alternatively, the time-domain relaxation data usually are fitted by means of the KWW stretched exponential function [2-5]

$\phi_{\mathrm{KWW}}(t)=\mathrm{e}^{-\left(\omega_{\mathrm{p}} t\right)^{\alpha}} \quad$ with $0<\alpha<1$.

The waiting-time representation (5) of the empirical functions (8) and (9) has been discussed 
Table 1

Probabilistic representation of the empirical responses

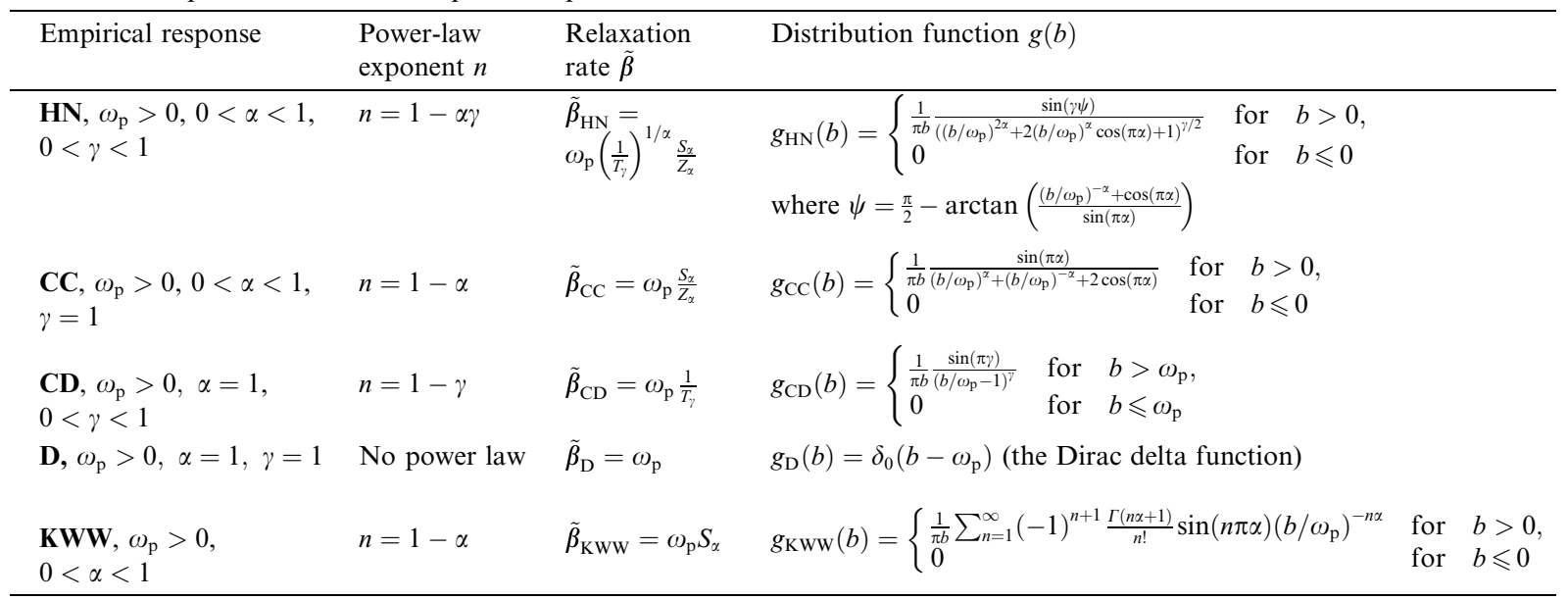

Random variable $T_{\gamma}$ is distributed according to the beta distribution with parameters $p=\gamma$ and $q=1-\gamma$ for $0<\gamma<1$ (called also the generalized arcsine distribution). $S_{\alpha}, Z_{\alpha}$ denote identically distributed Lévy-stable random variables defined by their Laplace transforms $\mathscr{L}\left(S_{\alpha}, t\right)=\mathscr{L}\left(Z_{\alpha}, t\right)=\exp \left(-t^{\alpha}\right), 0<\alpha<1$. All the random variables are (stochastically) independent.

recently; see [26] where the distributions of the corresponding waiting times $\tilde{\theta}$, as well as their properties, are derived in details. Here, in Table 1 we present the relaxation-rate representation (6) of the functions together with the corresponding probability density functions $g(b)$. Let us mention that the relaxation-time density functions $w(\tau)$ which produce the empirical non-exponential KWW and $\mathrm{HN}$ responses have been known for almost three decades [2,30]. However, this information has given only slight progress in clarifying the underlying physical mechanism; and the socalled distribution-function approach has been used mainly as a formal mathematical tool convenient to describe, analyze, and transform the data in order to compare them with the results obtained by different experimental techniques, see e.g. [31-33]. The reason for this is that $\phi(t)$, defined by Eq. (3), is a function describing the relaxation process of the system as a whole; and the approach concerns, in fact, the effective behavior of the macroscopic system represented by one (real or imaginary) object with the value of relaxation time $\tau$ randomly taken according to the distribution of $\tilde{T}$. In order to make a progress in clarifying the relaxation phenomenon, one should use mathematical tools capable of relating the relaxational properties of the structural elements to the effective representation of the system.

\section{Microscopic stochastic scenario of relaxation}

In any dielectric complex system capable of responding to an external electric field it is possible that only a part of the total number $N$ of dipoles in the system is able to follow significantly changes of the field [5]. In general, the distribution of the random waiting time $\tilde{\theta}$ of the entire system is determined by the first passage of the system from its initial state $[11,24,25]$. It can be expressed in the following form:

$\operatorname{Pr}(\tilde{\theta} \geqslant t)=\operatorname{Pr}\left(\min \left(\theta_{1 N}, \ldots, \theta_{v_{N} N}\right) \geqslant t\right)$,

where $N$ denotes the size of the system, $v_{N}$ is a number of the dipoles taking essentially part in the relaxation process, and variables $\theta_{i N}\left(i=1, \ldots, v_{N}\right)$ represent the random waiting times of the particular responding dipoles for the initial state transition.

As shown in the previous chapter, the system waiting time $\tilde{\theta}$ is assigned to the system relaxation rate $\tilde{\beta}$ by means of (7). It is natural to assume the 
similar relation between the individual waiting times $\theta_{i N}$ and individual relaxation rates, say $\beta_{i N}$, of the 'active' responding entities in the system; namely,

$\operatorname{Pr}\left(\theta_{i N} \geqslant t\right)=\left\langle\mathrm{e}^{-t \beta_{i N}}\right\rangle$.

According to the rate-theory concept, the individual relaxation rates $\beta_{1 N}, \beta_{2 N}, \ldots$ are considered here as the contributions of the dipoles to the total relaxation rate $\tilde{\beta}$, see e.g. [34]. Properties of the individual relaxation rates reflect the influence of the medium and those dipoles that do not contribute directly to the relaxation dynamics, but may affect the stochastic transition of the active entities.

The non-negative waiting times $\theta_{1 N}, \theta_{2 N}, \ldots$ and relaxation rates $\beta_{1 N}, \beta_{2 N}, \ldots$ are assumed to form sequences of independent and identically distributed random variables. As a consequence, we have

$\phi(t)=\operatorname{Pr}(\tilde{\theta} \geqslant t)=\prod_{i=1}^{v_{N}} \operatorname{Pr}\left(\theta_{i N} \geqslant t\right)$

in accordance with the definitions of the relaxation function introduced in other probabilistic approaches to relaxation, see e.g. [6,34]. Then, applying relation (10), we obtain that the relaxation function (5) can be expressed as

$\phi(t)=\prod_{i=1}^{v_{N}}\left\langle\mathrm{e}^{-t \beta_{i N}}\right\rangle=\left\langle\mathrm{e}^{-t \tilde{\beta}}\right\rangle$,

where $\tilde{\beta}$ has a form of the sum of the individual relaxation rates of all responding dipoles

$\tilde{\beta}=\sum_{i=1}^{v_{N}} \beta_{i N}$.

It should be noted $[24,25]$ that we can come to the same conclusion even if the number $v_{N}$ of the active responding dipoles is not a deterministic number but an integer-valued random variable (independent of the waiting times $\theta_{i N}$ and relaxation rates $\beta_{i N}$ ) the distribution of which depends on the system size $N$.

In order to find $v_{N}$, let us assume first that the system is divided into clusters of sizes $N_{1}, N_{2}, \ldots$, determined by the local interactions. The number $K_{N}$ of the clusters is equal to the first index $k$ for which the sum $N_{1}+\cdots+N_{k}$ of the cluster sizes exceeds $N$, the size of the system. ${ }^{1}$ Mathematically, one can formulate this as follows:

$K_{N}=\min \left\{k: \sum_{i=1}^{k} N_{i}>N\right\}$.

Depending on the screening mechanisms [3], the active responding dipoles may 'see' some of their active neighbours. If so, the cooperative regions built up from the responding dipoles may appear. The contribution of each region to the total relaxation rate is the sum of the contributions of all active dipoles over the region. Hence, for the $j$ th region containing $M_{j}$ interacting active dipoles its relaxation rate, say $\overline{\beta_{j N}}$, is equal to

$\overline{\beta_{j N}}=\sum_{i=M_{1}+\cdots+M_{j-1}+1}^{M_{1}+\cdots+M_{j}} \beta_{i N}$.

For $j=1$ it is simply the sum $\overline{\beta_{1 N}}=\sum_{i=1}^{M_{1}} \beta_{i N}$; for $j=2$ it is $\overline{\beta_{2 N}}=\sum_{i=M_{1}+1}^{M_{1}+M_{2}} \beta_{i N}$, and so on.

Following (6), the effective representation of the system as a whole, namely, $\phi(t)=\left\langle\mathrm{e}^{-t \tilde{\beta}_{N}}\right\rangle$, is provided by the total relaxation rate $\tilde{\beta}_{N}$ that is the sum of the contributions over all cooperative regions. Since the number $L_{N}$ of such mesoscopic regions in the system consisting of $K_{N}$ clusters is determined by the cooperative-regions sizes $M_{1}, M_{2}, \ldots$ and equals ${ }^{2}$

$L_{N}=\min \left\{l: \sum_{j=1}^{l} M_{j}>K_{N}\right\}$,

we have

$\tilde{\beta}_{N}=\sum_{j=1}^{L_{N}} \overline{\beta_{j N}}$.

The relaxation rate $\tilde{\beta}_{N}$ of the form (14) can be easily rewritten into the form (11) leading to the explicit formula

\footnotetext{
${ }^{1}$ (The example) Let the system size be $N=100$. Let the values of the cluster sizes be $N_{1}=N_{5}=14, N_{2}=N_{6}=12$, $N_{3}=13, N_{4}=N_{8}=11, N_{7}=16$. Since $N_{1}+\cdots+N_{7}=92<$ $N=100$ and $N_{1}+\cdots+N_{7}+N_{8}=103>N=100$, we obtain $K_{N}=8$.

${ }^{2}$ (The example, continued) Let the values of the region sizes be $M_{1}=M_{2}=3, M_{3}=4$. Since $M_{1}+M_{2}=6<K_{N}=8$ and $M_{1}+M_{2}+M_{3}=10>K_{N}=8$, we obtain $L_{N}=3$ and $v_{N}=10$.
} 
$v_{N}=\sum_{j=1}^{L_{N}} M_{j}$

for the number $v_{N}$ of the dipoles which are able to follow changes of the field in the system built up of the dynamically correlated regions.

Let us add that the individual relaxation rates can be assumed to take the form $\beta_{i N}=\beta_{i} / A_{N}$ with $\beta_{i}$ independent of the system size $N$ and the same normalizing constant $A_{N}$ for each dipole. In this case,

$\tilde{\beta}_{N}=\frac{1}{A_{N}} \sum_{i=1}^{v_{N}} \beta_{i}$.

It follows from the physical intuition that, in general, the number of dipoles directly engaged in the relaxation process, as well as their locations, are random. Therefore, not only $\beta_{i N}$ but also all the quantities $N_{i}, M_{j}$, and those defined by them, have to be considered as random variables. Their stochastic characteristics would obviously determine the properties of the total relaxation rate $\tilde{\beta}_{N}$ if they were known. But they are not, unfortunately. Nevertheless, studying relaxation phenomena, one usually deals with systems consisting of a large number of dipoles. Therefore, due to the form (16) of the relationship between the relaxation rates of the constituents and the total rate of the system, we are able to use the powerful tool of limit theorems of probability theory for the analysis of the relaxation mechanism. On the basis of limit theorems, it is possible to define the distribution of the limit

$\tilde{\beta}=\lim _{N \rightarrow \infty} \tilde{\beta}_{N}=\lim _{N \rightarrow \infty} \frac{1}{A_{N}} \sum_{i=1}^{v_{N}} \beta_{i}$

even if the distributions of micro/mesoscopic quantities are known in a relatively limited extent only. In large relaxing systems the limit $\tilde{\beta}$ can represent the entire system in (6) instead of the exact $\tilde{\beta}_{N}$ (in practice, it is enough when $N \sim 10^{6}$ ).

In the proposed scheme we take stochastically independent sequences of random variables $N_{i}, M_{j}$, and $\beta_{i}$. Each sequence consists of independent and identically distributed non-negative random variables that have either finite expected value or heavy-tailed distribution. Then, if the normalizing

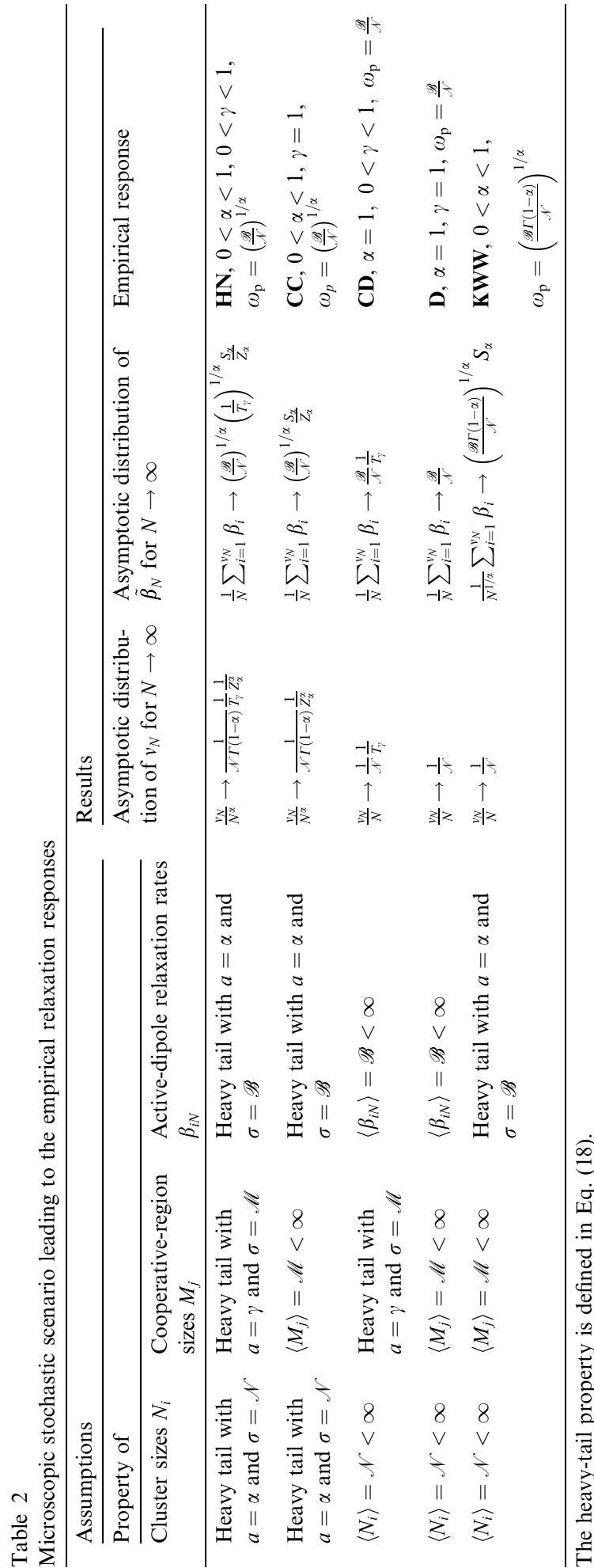



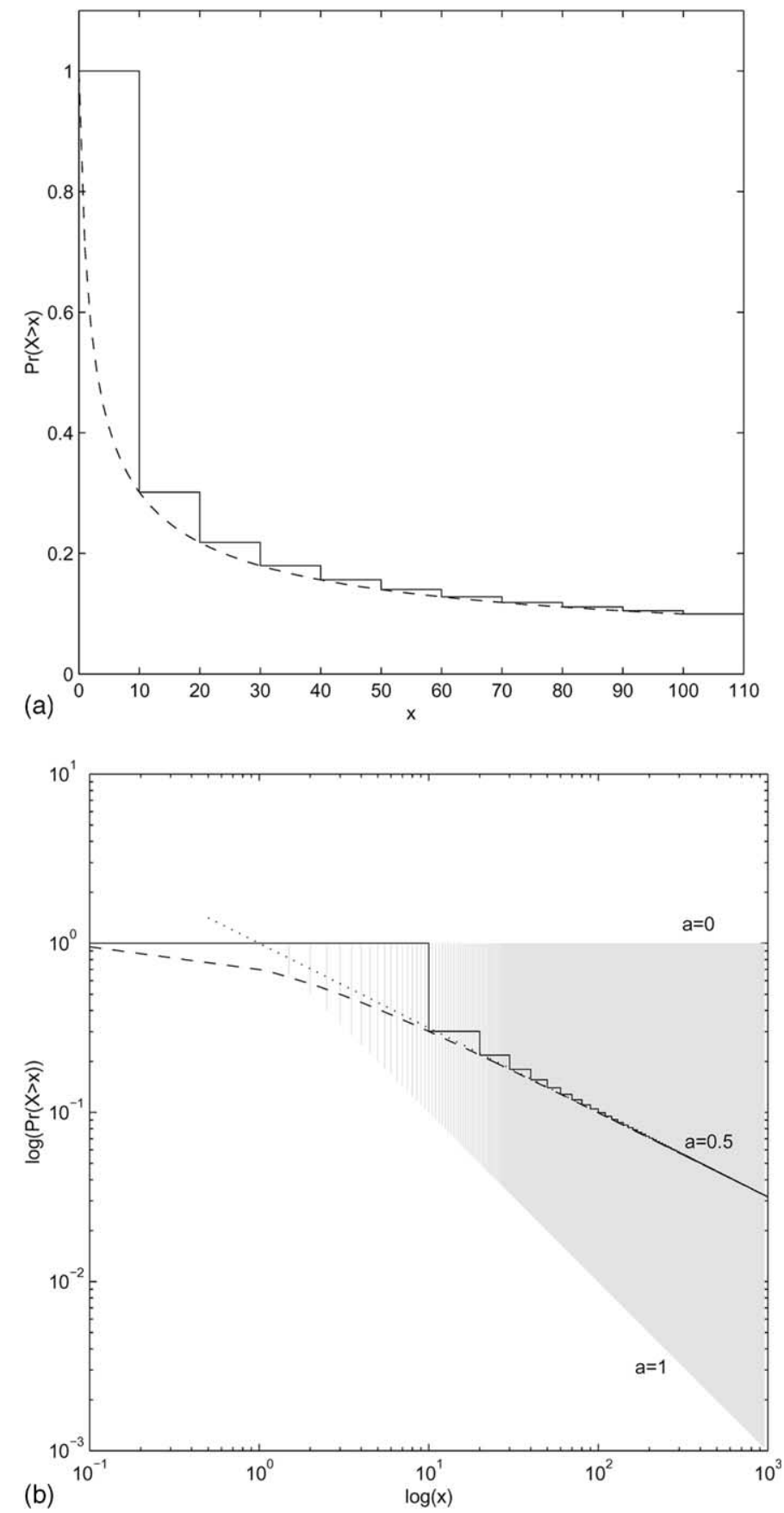

Fig. 1. Examples of continuous and discrete heavy-tailed distributions. (a) The tail functions of the continuous heavy-tailed Pareto random variable $X$ with $A=1$ and $b=0.5(--)$ and of the discrete random variable $X_{\mathrm{d}}$ such that $X_{\mathrm{d}}=10 n$ if and only if $10(n-1)<X<10 n$ for $n=1,2, \ldots$ (-). The heavy-tailed Pareto distribution is defined by $\operatorname{Pr}(X>x)=(1+A x)^{-b}$ with $A>0$ and $0<b<1$. The discrete random variable $X_{\mathrm{d}}$ is obtained from $X$ by the quantizer transformation. For both cases condition (18) is satisfied with $a=b$. (b) The $\log -\log$ plot of the tail function $\operatorname{Pr}(X>x)$ shows if the distribution of random variable $X$ has a heavy tail. The heavy-tail property manifests there as the asymptote $(\cdots)$ of the plotted function with the slope parameter $-a$ between -1 and 0 (that is, situated in the marked area). 
constants $A_{N}$ in (11) are chosen properly, the total relaxation rate $\tilde{\beta}$ in (17) takes the form corresponding to one of the empirical responses; for details see Table 2 in comparison with Table 1. Under the same assumptions, the asymptotic distribution of $v_{N}$ given by (15) can be derived as mentioned in Table 2. It is worth noting here that, although $v_{N}$ is always greater than $K_{N}$, the number of clusters in the system (given by (12), their asymptotic properties for $N \rightarrow \infty$ are the same in the cases corresponding to the $\mathrm{CC}, \mathrm{D}$, and KWW responses. The significant difference resulting from the heavy-tail property of the cooperative-region size distribution appears, however, in the HN and CD cases.

Let us add that the distribution of a nonnegative random variable, say $X$, has a heavy tail if the tail $\operatorname{Pr}(X>x)$ satisfies the condition

$\lim _{x \rightarrow \infty} \frac{\operatorname{Pr}(X>x)}{\sigma x^{-a}}=1$

for some $0<a<1$ and $\sigma>0$; that is, if for large values of $x$ the tail exhibits a fractional power law $\sigma x^{-a}$ (see also Fig. 1). There are many different continuous and discrete distributions satisfying condition (18). Classical examples of continuous ones are completely asymmetric Lévy-stable laws, also the Pareto and Burr distributions with an appropriate choice of their parameters $[35,36]$. To get discrete distributions with heavy tails, one can simply apply a quantizer transformation [37] to some of the above continuous examples as shown in Fig. 1.

If the distribution of random variable $X$ has a heavy tail, then the expected value $\langle X\rangle$ is infinite. Therefore, the two considered attributes, the finiteness of the expected value and the heavy-tail property (18), clearly exclude each other. Besides, both provide only limited information on the corresponding distributions. Hence, the conditions put on the distributions of the microscopic quantities in the proposed scheme (see Table 2) are rather general. On the other hand, the macroscopic result is determined in any detail.

\section{Conclusions}

In this paper we have proposed a probabilistic approach to model non-exponential relaxation processes which leads to the well-known empirical laws. In this approach, going beyond the classical methods of statistical physics, the link between the microscopic world of real objects forming the system and the macroscopic world of physical phenomena is provided by the internal structure of the total random relaxation rate. The form of the total rate follows from the mathematical construction of an imaginary object representing the relaxation behavior of the entire complex system the construction which is based on the hierarchical dynamics in the multichannel scheme of relaxation

Table 3

Discussion of the energy criterion on the micro/meso/macroscopic levels

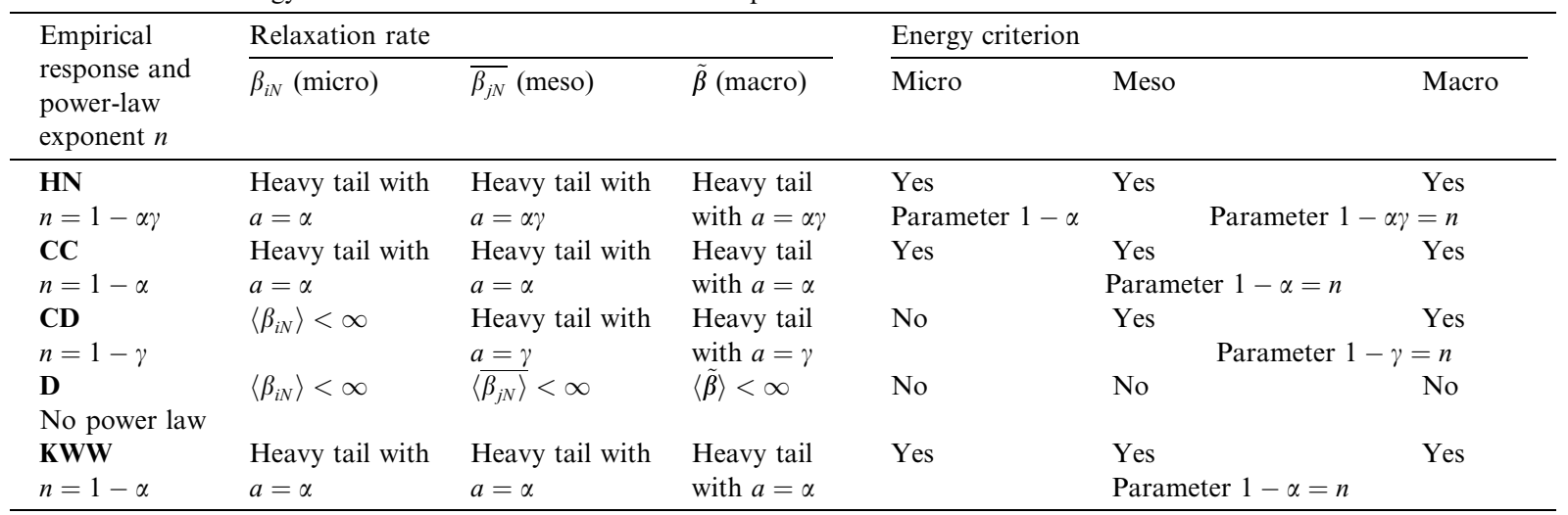


and the idea of limit theorems of probability theory. The explicit effective representation of the system, expressed in terms of the total relaxation rate, has been then shown to be related to the phenomenological $\mathrm{HN}$ and KWW laws, given by (8) and (9), under general conditions put on the stochastic properties of the system constituents, see Table 2.

If the model yields one of the macroscopic responses exhibiting the high-frequency power law (1) with the exponent $n$ (i.e. to $\mathrm{HN}, \mathrm{CC}, \mathrm{CD}$ or KWW response), the distribution of at least one of the microscopic quantities $N_{i}, M_{j}, \beta_{i}$ has a heavy tail. In such a case both, the distribution of cooperative-region relaxation rates $\overline{\beta_{j N}}$ and the distribution of the total relaxation rate $\tilde{\beta}$, also satisfy condition (18), with the same parameter $a=1-n$, see Table 3. Condition (18) applied to any relaxation rate $\beta$ can be expressed in the form

$\operatorname{Pr}(\beta \geqslant b / c) \approx c^{a} \operatorname{Pr}(\beta \geqslant b), \quad b \rightarrow \infty$

(for any fixed constant $c>0$ ) that reflects the scaling property of the rate. It can be shown [38] that this property corresponds to the highfrequency power-law asymptotic behavior of the associated susceptibility $\chi(\omega)$ with the power-law exponent equal to $a$ and leads to the relation

$\frac{\chi^{\prime \prime}(\omega)}{\chi^{\prime}(\omega)}=\cot \left((1-a) \frac{\pi}{2}\right)$ for $\omega \gg \omega_{\mathrm{p}}$,

interpreted as the energy criterion. Hence, the scaling property (19) of micro/meso/macroscopic relaxation rate with the exponent $a$ leads to the corresponding micro/meso/macroscopic constant ratio (20) with the parameter $1-a$.

The relation (20) is consistent with the energy criterion (2) if only $1-a$ is equal to the macroscopic high-frequency power-law exponent $n$. On the mesoscopic level this holds in all cases corresponding to the non-exponential responses. In contrast, the distribution of the active-dipole relaxation rate $\beta_{i N}$ has a heavy tail in case of the $\mathrm{HN}$, $\mathrm{CC}$, and KWW responses only. In each mentioned case, the constant ratio of the imaginary to the real parts of the microscopic complex susceptibility

$\frac{\chi_{i}^{\prime \prime}(\omega)}{\chi_{i}^{\prime}(\omega)}=\cot \left((1-\alpha) \frac{\pi}{2}\right)$ for $\omega \gg \omega_{\mathrm{p}}$, can be derived. However, the characteristic constant $1-\alpha$ does not fully determine the macroscopic exponent $n$ of the $\mathrm{HN}$ response where the influence of the cooperative-region size distribution appears as well. In the CD case the origins of the macroscopic energy criterion different than those indicated by the microscopic criterion are found. The high-frequency power law of this response results only from the heavy-tail property of the distribution of cooperative-region sizes, see Table 2.

The above considerations confirm the Jonscher's hypothesis that the identical property of individual structural elements of the system is hidden behind the macroscopic energy criterion (2); it appears, however, that this hypothesis should concern the mesoscopic cooperative regions rather than the particular responding dipoles themselves. The presented approach justifies the applicability of the interpretation proposed by Jonscher on the micro/meso/macroscopic levels, and reveals the self-similar dynamics hidden in the energy-criterion idea. We hope that the discussion of the energy criterion as a natural and inevitable physical condition for observation of property (1) in relaxation phenomena may open a new line of understanding these phenomena.

\section{References}

[1] A.K. Jonscher, Nature 267 (1977) 673.

[2] C.J. Bőttcher, P. Bordewijk, Theory of Electronic Polarization, vol. 2, Elsevier, Amsterdam, 1978.

[3] A.K. Jonscher, Dielectric Relaxation in Solids, Chelsea Dielectrics, London, 1983.

[4] S. Havriliak Jr., S.J. Havriliak, J. Non-Cryst. Solids 172174 (1994) 297.

[5] A.K. Jonscher, Universal Relaxation Law, Chelsea Dielectrics Press, London, 1996.

[6] J. Klafter, M.F. Shlesinger, Proc. Nat. Acad. Sci. USA 83 (1986) 848.

[7] L.A. Dissado, R.M. Hill, Chem. Phys. 111 (1987) 193.

[8] M.R. de la Fuente, M.A. Perez Jubindo, J.D. Solier, M.J. Tello, Phys. Rev. B 37 (1988) 2094.

[9] R.V. Chamberlin, D.N. Haines, Phys. Rev. Lett. 65 (1990) 2197.

[10] K.L. Ngai, R.W. Rendell, D.J. Plazek, J. Chem. Phys. 94 (1991) 3018.

[11] K. Weron, A. Jurlewicz, J. Phys. A 26 (1993) 395.

[12] V. Halpern, J. Phys.: Condens. Matter 7 (1995) 7687.

[13] I. Koponen, J. Non-Cryst. Solids 189 (1995) 154. 
[14] R.R. Nigmatullin, Ya.E. Ryabov, Phys. Solid State 39 (1997) 87.

[15] R. Metzler, E. Barkai, J. Klafter, Phys. Rev. Lett. 82 (1999) 3563.

[16] E. Donth, J. Non-Cryst. Solids 53 (1982) 325.

[17] C. Schick, E. Donth, Phys. Scr. 43 (1991) 423.

[18] C.T. Moynihan, J. Schroeder, J. Non-Cryst. Solids 160 (1993) 52.

[19] M.T. Cicerone, M.D. Ediger, J. Chem. Phys. 103 (1995) 5684.

[20] K. Schmidt-Rohr, H.W. Spiess, Phys. Rev. Lett. 66 (1991) 3020 .

[21] A. Heuer, M. Wilhelm, H. Zimmermann, H.W. Spiess, Phys. Rev. Lett. 75 (1995) 2851.

[22] B. Schiener, R. Bohmer, A. Loidl, R.V. Chamberlin, Science 274 (1996) 752.

[23] R.V. Chamberlin, Europhys. Lett. 33 (1996) 545.

[24] K. Weron, M. Kotulski, J. Stat. Phys. 88 (1997) 1241.

[25] A. Jurlewicz, K. Weron, Cell. Molec. Biol. Lett. 4 (1999) 55.

[26] A. Jurlewicz, K. Weron, Acta Phys. Pol. B 31 (2000) 1077.

[27] S. Bovelli, D. Fioretto, A. Jurlewicz, J. Phys.: Condens. Matter 13 (2001) 373.
[28] K. Weron, A. Jurlewicz, IEEE Trans. Dielectrics and E.I. 8 (2001) 352.

[29] N.G. Van Kampen, Stochastic Processes in Physics and Chemistry, North Holland, Amsterdam, 1987.

[30] J.V. Bertelsen, A. Lindgård, J. Polym. Sci. 12 (1974) 1707.

[31] C.P. Lindsey, G.P. Patterson, J. Chem. Phys. 73 (1980) 3348.

[32] F. Alvarez, A. Alegría, J. Colmenero, Phys. Rev. B 47 (1993) 125.

[33] F. Alvarez, A. Alegría, J. Colmenero, J. Chem. Phys. 103 (1995) 798.

[34] M.O. Vlad, M.C. Mackey, J. Math. Phys. 36 (1995) 1834.

[35] W. Feller, An Introduction to Probability Theory and Its Applications, vols. 1\&2, Wiley, New York, 1966.

[36] N.L. Johnson, S. Kotz, Distributions in Statistics: Discrete Distributions; Distributions in Statistics: Continuous Univariate Distributions 1, 2, Wiley, New York, 1969\&1970.

[37] Y. Viniotis, Probability and Random Processes for Electrical Engineers, McGraw-Hill, Boston, 1998.

[38] A.I. Saichev, W.A. Woyczynski, Distributions in the Physical and Engineering Sciences, Birkhäuser, Boston, MA, 1997. 